\title{
Evaluation of Ocular Biomechanical Indices to Distinguish Normal from Keratoconus Eyes
}

\author{
Allan Luz, Bruno Fontes, Isaac C Ramos, Bernardo Lopes, Fernando Correia, Paulo Schor, Renato Ambrósio Jr
}

\begin{abstract}
Purpose: To compare and assess the ability of pressure-derived parameters and corneal deformation waveform signal-derived parameters of the ocular response analyzer (ORA) measurement to distinguish between keratoconus and normal eyes, and to develop a combined parameter to optimize the diagnosis of keratoconus.
\end{abstract}

Materials and methods: One hundred and seventy-seven eyes (177 patients) with keratoconus (group KC) and 205 normal eyes (205 patients; group N) were included. O ne eye from each subject was randomly selected for analysis. Patients underwent a complete clinical eye examination, corneal topography (Humphrey ATLAS), tomography (Pentacam Oculus) and biomechanical evaluations (ORA Reichert). Differences in the distributions between the groups were assessed using the MannWhitney test. The receiver operating characteristic (ROC) curve was used to identify cutoff points that maximized sensitivity and specificity in discriminating keratoconus from normal corneas. Logistic regression was used to identify a combined linear model (Fisher 1.0)

Results: Significant differences in all studied parameters were detected $(p<0.05)$, except for W2. For the corneal resistance factor (CRF): Area under the ROC curve (AUROC) $89.1 \%$, sensitivity $81.36 \%$, specificity $84.88 \%$. F or the plarea: AUROC $91.5 \%$, sensitivity $87.1 \%$, specificity $81.95 \%$. Of the individual parameters, the highest predictive accuracy was for the Fisher 1.0 , which represents the combination of all parameters (AUROC $95.5 \%$, sensitivity $88.14 \%$, specificity $93.17 \%$ ).

Conclusion: Waveform-derived ORA parameters displayed greater accuracy than pressure-derived parameters for identifying keratoconus. Corneal hysteresis $(\mathrm{CH})$ and $\mathrm{CRF}, \mathrm{a}$ diagnostic linear model that combines different parameters, provided the greatest accuracy for differentiating keratoconus from normal corneas.

Keywords: Keratoconus, Cornea, Biomechanical indices, Applanation, Waveform.

How to cite this article: Luz A, Fontes B, Ramos IC, Lopes B, Correia F, Schor P, Ambrósio R. Evaluation of Ocular Biomechanical Indices to Distinguish Normal from Keratoconus Eyes. Int J Kerat Ect Cor Dis 2012;1(3):145-150.

\section{Source of support: $\mathrm{Nil}$}

Conflict of interest: None declared

\section{INTRODUCTION}

The cornea is a tissue with viscoelastic properties. A lterations in its stromal structure are intimately related to its biomechanical behavior. ${ }^{1}$ The commercial ocular response analyzer (ORA ; R eichert O phthalmic Instruments, Depew, NY, USA) utilizes a dynamic bidirectional applanation process to quantify corneal biomechanical properties in vivo and to determine intraocular pressure $(I O P)^{2}$

There has been increased interest in evaluating corneal biomechanics. The ORA records corneal inward and outward applanation after delivering a metered collimated air pulse and provides an indication of the viscosity and elastic properties of the cornea. Corneal hysteresis $(\mathrm{CH})$ and the corneal resistance factor (CRF), which are the corneal biomechanical metrics generated by the ORA, have been the subjects of several recent publications. ${ }^{3,4}$

Luce presented data ( $L$ uce D, ORA waveform analysis and beyond. Presented at: A merican Society of Cataract and Refractive Surgery A nnual Meeting, A pril 3 to 8, 2009; San Francisco, California) indicating that waveform parameters provided from the ORA signal may be more sensitive than $\mathrm{CH}$ or the $\mathrm{CRF}$ in discriminating abnormal corneas. The differences in the signal morphology between the two eyes led to the conclusion that the corneas are biomechanically distinct. ${ }^{5}$

The purpose of this study was to evaluate and compare the ORA parameters between patients with keratoconus and healthy control individuals. In addition, we assessed the effect of analysis of all ORA parameters together.

\section{MATERIALS AND METHODS}

The study constituted a comparative case series. The research followed the tenets of the Declaration of H elsinki and was approved by the ethics committee of the Federal University of São Paulo, Brazil (protocol 2012/10). The purpose of the study was explained to all subjects, who gave informed consent before inclusion. Patients were evaluated sequentially from D ecember, 2010 through D ecember, 2011. Demographic and clinical data were obtained, including date of birth, gender and self-reported race or ethnicity.

Each subject underwent a comprehensive ophthal mologic examination, which included a medical history review, best corrected visual acuity, slit-lamp and funduscopic examinations, Placido disc topography (Humphrey A TLAS; Carl Zeiss Meditec, Dublin, CA, USA), Pentacam tomographic evaluation (Oculus, W etzlar, Germany), and ORA measurements (Reichert Ophthalmic Instruments).

Keratoconus was defined using the A msler-K rumeich classification. ${ }^{6} \mathrm{~A}$ normal eye had no ocular pathology, 
previous ocular surgery or relevant refractive error. One eye randomly selected from 177 consecutive patients with clinical bilateral keratoconus was retrospectively included (group KC). The control group included one eye randomly selected from 205 age-matched patients from a database of normal patients considered good candidates (group N). Keratoconus cases with a history of corneal surgery or with extensive corneal scarring were excluded from the study.

A ccording to the A msler- $K$ rumeich classification of the severity of keratoconus, 120 eyes (67.8\%) were classified as grade I, $37(20.9 \%)$ as grade II and $20(11.3 \%)$ as grade III.

The ORA determines corneal biomechanical properties using an applied force displacement relationship. A precisely metered air pulse is delivered to the eye, causing the cornea to move inward, past applanation and into slight concavity. Milliseconds after the initial applanation, the air pump generating the air pulse is shut off, and the pressure applied to the eye decreases in an inverse time, symmetrical fashion. As the pressure decreases, the cornea passes through a second applanated state while returning from concavity to its normal convex curvature. Energy absorption during rapid corneal deformation delays the occurrence of the inward and outward applanation signal peaks, resulting in a difference between the applanation pressures. This difference between the inward and outward motion applanation pressures is $\mathrm{CH}$, which indicates viscous damping in the cornea and reflects the capacity of corneal tissue to absorb and dissipate energy. The CRF is a measure of the cumulative effects of both the viscous and elastic resistance encountered by the air jet while deforming the corneal surface, being an indicator of the overall resistance of the cornea. The CRF was derived empirically to maximize its correlation with the central corneal thickness. It can be considered as weighted by the elastic resistance, because of its stronger correlation with the central corneal thickness than with $\mathrm{CH}$. Although $\mathrm{CH}$ and the $\mathrm{CRF}$ are related, they can in some cases differ significantly, and each provides distinct information about the cornea.

Using the new ORA software (version 3.00), 37 new parameters were calculated based on the waveform of the ORA signal. Six further keratoconus-specific parameters are incorporated in the latest update to the ORA device. These are: The keratoconus match index (K M I-KC score), and the keratoconus match probability (KM P-KC normal, $\mathrm{K}$ suspect, $\mathrm{KC}$ mild, $\mathrm{KC}$ moderate and $\mathrm{K} C$ severe).

$L$ inear discriminant analysis (LDA), and the related Fisher's linear discriminant (Fisher 1.0), are methods used in statistics, pattern recognition and machine learning to identify a linear combination of features that characterizes or separates two or more classes of objects or events. The resulting combination may be used as a linear classifier or, more commonly, for dimensionality reduction before later classification.

Statistical analyses were performed using B ioEstat 5.0 (Instituto M amirauá, A mazonas, B razil) and M ed-Calc 11.1 (MedCalc Software, M ariakerke, Belgium). The nonparametric $M$ ann-W hitney $U$ test (W ilcoxon rank-sum test) was used to assess variable distributions between the keratoconic and normal cornea groups.

Receiver operating characteristic (ROC) curves and the areas under the ROC curves (AUROCS) were calculated for all parameters to determine the overall predictive accuracy of the tests. The standard error of the AUROC was assessed by the DeL ong method. ${ }^{7}$ The binomial exact method was used to calculate the confidence interval $(\mathrm{Cl})$ for the A UR OC. N onparametric pairwise comparisons were performed to determine the significance of differences between AUROCs, using the Hanley-M CNeil method ${ }^{8}$ to calculate standard errors. Values of $p<0.05$ indicated statistical significance.

\section{RESULTS}

Single eyes randomly selected from 205 patients with normal, unoperated eyes and 177 patients with bilateral keratoconus were included. In the normal and keratoconic groups, the average patient ages were $34.0 \pm 10.9$ years (range: $12.0-78.1$ years) and $30.2 \pm 10.8$ years (range: 16.1 63.0 years) respectively.

Significant differences were found between normal (group N) and keratoconic (group KC) eyes for all parameters ( $M$ ann-W hitney $U$ test, $p<0.05$ ) with the exception of the w2 parameter ( $p=0.0491$; Table 1$)$. $\mathrm{CH}$ was $8.36 \pm 1.63 \mathrm{~mm} \mathrm{Hg}$ (range: $13.60-3.90 \mathrm{~mm} \mathrm{Hg}$ ) in group $\mathrm{KC}$ and $10.66 \pm 1.71 \mathrm{~mm} \mathrm{Hg}$ (range: 15.90-6.20) in group $N(p<0.0001)$. The corneal resistance factor was $7.35 \pm$ $2.05 \mathrm{~mm} \mathrm{Hg}$ (range: $20.90-3.00$ ) in group $\mathrm{KC}$ and $10.56 \pm$ $1.98 \mathrm{~mm} \mathrm{Hg}$ (range: $16.10-6.00)$ in group $\mathrm{N}(\mathrm{p}<0.0001)$. The 1 area was 1,938.32 \pm 869.33 (range: 4,849.13-278.19) in group KC and 3,864.45 $\pm 1,231.35$ (range: $10,147.64$ $1,382.00)$ in group $N(p<0.0001)$. The KMI (KC score) was $0.07309 \pm 0.379904$ (range: 2.043 to -0.0747 ) in group $K C$ and $0.805224 \pm 0.394059$ (range: 1.806 to -0.111 ) in the group $N(p<0.0001)$. The KMP (KC normal) was $5.870056 \pm 15.92811$ (range: 100 to 0 ) in group KC and $50.37561 \pm 37.55337$ (range: 100 to 0 ) in group $N$ ( $p<$ 0.0001 ). The Fisher 1.0 was $-0.90739 \pm 0.40433$ (range: 0.862008 to -2.16366 ) in group $K C$ and $0.057053 \pm$ 0.402856 (range: 1.079689 to -1.2191 ) in group $N$. These data are summarized in Table 1.

A II parameters had higher values in group $\mathrm{N}$ with the exception of path $1,2,11$, and 21 , aplhf and KMP 
Evaluation of Ocular Biomechanical Indices to Distinguish Normal from Keratoconus Eyes

Table 1: ORA parameters measured in normal and keratoconus eyes

\begin{tabular}{|c|c|c|c|c|c|c|c|c|c|}
\hline & \multicolumn{4}{|c|}{$N L$} & \multirow[b]{2}{*}{$p$-value } & \multicolumn{4}{|c|}{$\mathrm{KC}$} \\
\hline & MED & DESVPAD & MAIOR & MENOR & & MED & DESVPAD & MAIOR & MENOR \\
\hline $\mathrm{IOPg}$ & 15.26 & 3.69 & 27.30 & 6.90 & $<0.0001$ & 11.09 & 4.09 & 41.20 & 3.10 \\
\hline IOPCC & 15.49 & 3.36 & 26.80 & 7.40 & $<0.0001$ & 14.38 & 3.55 & 36.60 & 3.90 \\
\hline CRF & 10.57 & 1.99 & 16.10 & 6.00 & $<0.0001$ & 7.36 & 2.06 & 20.90 & 3.00 \\
\hline $\mathrm{CH}$ & 10.66 & 1.71 & 15.90 & 6.20 & $<0.0001$ & 8.37 & 1.63 & 13.70 & 3.90 \\
\hline KC score & 0.81 & 0.39 & 1.81 & -0.11 & $<0.0001$ & 0.07 & 0.38 & 2.04 & -0.75 \\
\hline KC normal & 50.38 & 37.55 & 100.00 & 0.00 & $<0.0001$ & 5.87 & 15.93 & 100.00 & 0.00 \\
\hline KC suspect & 32.37 & 24.54 & 68.00 & 0.00 & $<0.0001$ & 20.10 & 21.75 & 68.00 & 0.00 \\
\hline $\mathrm{KC}$ mild & 15.13 & 17.64 & 65.00 & 0.00 & $<0.0001$ & 36.30 & 18.89 & 65.00 & 0.00 \\
\hline $\mathrm{KC}$ moderate & 2.00 & 5.99 & 48.00 & 0.00 & $<0.0001$ & 25.35 & 19.96 & 57.00 & 0.00 \\
\hline $\mathrm{KC}$ severe & 0.13 & 0.85 & 9.00 & 0.00 & $<0.0001$ & 12.38 & 20.07 & 94.00 & 0.00 \\
\hline aindex & 9.20 & 1.05 & 10.00 & 5.35 & $<0.0001$ & 7.65 & 2.39 & 10.00 & 1.18 \\
\hline bindex & 9.43 & 1.11 & 10.00 & 0.48 & $<0.0001$ & 8.04 & 2.59 & 10.00 & 0.21 \\
\hline plarea & 3864.45 & 1231.35 & 10147.64 & 1382.00 & $<0.0001$ & 1938.32 & 869.33 & 4849.13 & 278.19 \\
\hline p2area & 2525.99 & 791.87 & 4610.50 & 762.31 & $<0.0001$ & 1268.27 & 687.44 & 6213.70 & 112.56 \\
\hline aspect1 & 19.49 & 5.90 & 39.31 & 7.77 & $<0.0001$ & 12.99 & 6.77 & 45.00 & 1.03 \\
\hline aspect2 & 20.40 & 9.33 & 55.02 & 4.52 & $<0.0001$ & 13.70 & 10.30 & 51.03 & 1.01 \\
\hline uslope1 & 66.28 & 29.40 & 187.17 & 17.64 & $<0.0001$ & 41.90 & 26.57 & 172.00 & 4.47 \\
\hline uslope2 & 88.89 & 41.46 & 239.13 & 14.68 & $<0.0001$ & 53.13 & 40.79 & 196.88 & 1.75 \\
\hline dslope1 & 28.81 & 8.67 & 60.83 & 11.55 & $<0.0001$ & 20.62 & 12.16 & 102.50 & 1.30 \\
\hline dslope2 & 27.28 & 13.75 & 84.80 & 5.59 & $<0.0001$ & 19.72 & 15.96 & 96.38 & 1.13 \\
\hline w1 & 21.90 & 2.62 & 30.00 & 15.00 & 0.00 & 20.68 & 5.64 & 43.00 & 6.00 \\
\hline w2 & 18.30 & 4.20 & 34.00 & 8.00 & 0.05 & 18.10 & 7.53 & 40.00 & 6.00 \\
\hline h1 & 418.61 & 110.03 & 651.00 & 171.00 & $<0.0001$ & 242.97 & 95.68 & 530.44 & 44.26 \\
\hline h2 & 344.40 & 107.01 & 615.56 & 147.00 & $<0.0001$ & 195.43 & 100.20 & 545.81 & 20.24 \\
\hline dive1 & 352.90 & 140.58 & 614.50 & 17.50 & $<0.0001$ & 204.05 & 100.76 & 516.00 & 9.25 \\
\hline dive2 & 276.28 & 108.57 & 552.75 & 25.00 & $<0.0001$ & 150.40 & 84.15 & 427.63 & 5.00 \\
\hline path1 & 21.94 & 3.81 & 36.44 & 10.08 & $<0.0001$ & 28.02 & 8.00 & 54.86 & 13.92 \\
\hline path2 & 25.60 & 6.46 & 56.18 & 11.57 & $<0.0001$ & 31.53 & 10.17 & 65.43 & 13.86 \\
\hline mslew1 & 112.80 & 39.99 & 239.50 & 40.75 & $<0.0001$ & 73.63 & 34.14 & 214.50 & 8.75 \\
\hline mslew2 & 133.36 & 54.52 & 332.75 & 25.75 & $<0.0001$ & 85.84 & 50.75 & 255.00 & 11.25 \\
\hline slew1 & 66.09 & 29.94 & 187.17 & 16.38 & $<0.0001$ & 46.08 & 26.12 & 172.00 & 4.63 \\
\hline slew2 & 88.90 & 41.45 & 239.13 & 12.50 & $<0.0001$ & 57.16 & 39.18 & 196.88 & 2.50 \\
\hline aplhf & 1.32 & 0.29 & 2.40 & 0.80 & $<0.0001$ & 1.68 & 0.45 & 3.80 & 0.90 \\
\hline plareal & 1665.86 & 629.61 & 5350.00 & 542.00 & $<0.0001$ & 800.14 & 395.48 & 2468.63 & 117.75 \\
\hline p2area1 & 1093.73 & 371.50 & 2142.25 & 240.88 & $<0.0001$ & 533.95 & 302.38 & 2663.28 & 28.22 \\
\hline aspect11 & 25.95 & 9.21 & 66.25 & 10.11 & $<0.0001$ & 19.10 & 10.64 & 63.84 & 1.02 \\
\hline aspect21 & 28.26 & 13.71 & 71.47 & 4.56 & $<0.0001$ & 19.63 & 14.68 & 85.06 & 1.88 \\
\hline uslope11 & 63.93 & 30.32 & 181.38 & 11.56 & $<0.0001$ & 44.51 & 27.00 & 164.38 & 4.75 \\
\hline uslope21 & 72.96 & 34.82 & 200.25 & 11.29 & $<0.0001$ & 47.77 & 35.95 & 177.50 & 0.00 \\
\hline dslope11 & 44.97 & 17.93 & 121.92 & 14.10 & $<0.0001$ & 34.21 & 20.07 & 110.50 & 1.23 \\
\hline dslope21 & 45.00 & 25.58 & 170.50 & 5.79 & $<0.0001$ & 32.57 & 25.66 & 127.00 & 2.43 \\
\hline w11 & 11.28 & 2.25 & 18.00 & 5.00 & $<0.0001$ & 9.87 & 3.66 & 29.00 & 4.00 \\
\hline w21 & 9.13 & 2.74 & 23.00 & 3.00 & $<0.0001$ & 8.16 & 3.53 & 22.00 & 3.00 \\
\hline h11 & 279.07 & 73.36 & 434.00 & 114.00 & $<0.0001$ & 161.98 & 63.78 & 353.63 & 29.51 \\
\hline h21 & 229.60 & 71.34 & 410.38 & 98.00 & $<0.0001$ & 130.29 & 66.80 & 363.88 & 13.49 \\
\hline path11 & 31.83 & 7.65 & 57.88 & 11.71 & $<0.0001$ & 39.54 & 11.23 & 69.00 & 16.70 \\
\hline path21 & 35.78 & 9.51 & 68.60 & 14.59 & $<0.0001$ & 42.14 & 13.20 & 86.81 & 17.11 \\
\hline Fisher1.0 & 0.06 & 0.40 & 1.08 & -1.22 & $<0.0001$ & -0.91 & 0.40 & 0.86 & -2.16 \\
\hline
\end{tabular}

Significant differences were found between normal and keratoconic eyes for all parameters (Mann-Whitney $U$ test, $p<0.05$ ) with the exception of $w 2(p=0.05)$.

( $\mathrm{KC}$ mild, $\mathrm{KC}$ moderate, and $\mathrm{KC}$ severe). Both the cornealcompensated IOP (IOPCC) and the Goldmann-correlated IOP $(\mathrm{OPg})$ were significantly different between the groups. Although the average group N IOPcC was $15.49 \mathrm{~mm} \mathrm{Hg}$ and that of group KC was $14.37 \mathrm{~mm} \mathrm{Hg}$, the difference was significant $(p<0.0001)$.

The AUROC was $>0.85$ for 11 parameters, including the CRF (0.891), but not $\mathrm{CH}(0.841)$. The parameters related to the area of the waveform during the second and first applanations gave similar KM I (KC score) and the KM P (KC normal) results of 0.915 and 0.910 respectively.

The parameter that achieved the best results was the Fisher 1.0, with AUROC 0.955 , and a sensitivity and specificity of 88.14 and $93.17 \%$ respectively. The sensitivity, specificity and AUROC results are shown in Table 2. In the comparison of those parameters with an AUROC $>0.85$, the Fisher 1.0 was significantly superior to all others (Table 3). 
Table 2: Data summary from ROC curve of new ORA parameters in normal and keratoconic eyes

\begin{tabular}{|c|c|c|c|c|c|}
\hline Parameters & Criterion & Sensitivity & Specificity & AUROC & IC $(95 \%)$ \\
\hline $\mathrm{IOPg}$ & 12.50 & 74.01 & 75.61 & 0.83 & $0.786-0.864$ \\
\hline IOPCC & 14.70 & 61.02 & 58.54 & 0.62 & $0.566-0.666$ \\
\hline CRF & 8.60 & 81.36 & 84.88 & 0.89 & $0.855-0.920$ \\
\hline $\mathrm{CH}$ & 9.40 & 80.23 & 74.63 & 0.84 & $0.800-0.876$ \\
\hline KC score & 0.341 & 81.36 & 88.29 & 0.92 & $0.882-0.941$ \\
\hline KC normal & 5.00 & 81.36 & 87.80 & 0.91 & $0.877-0.937$ \\
\hline KC suspect & 14.00 & 58.19 & 66.34 & 0.64 & $0.587-0.685$ \\
\hline $\mathrm{KC}$ mild & 18.00 & 81.36 & 70.73 & 0.81 & $0.764-0.846$ \\
\hline $\mathrm{KC}$ moderate & 3.00 & 81.36 & 87.80 & 0.90 & $0.868-0.930$ \\
\hline $\mathrm{KC}$ severe & 0.00 & 61.58 & 96.10 & 0.80 & $0.752-0.835$ \\
\hline aindex & 9081.00 & 61.58 & 70.24 & 0.70 & $0.648-0.742$ \\
\hline bindex & 9708.00 & 59.32 & 60.00 & 0.65 & $0.602-0.700$ \\
\hline plarea & 2814789.00 & 87.01 & 81.95 & 0.92 & $0.882-0.941$ \\
\hline p2area & 1626.375 & 80.23 & 88.29 & 0.91 & $0.876-0.936$ \\
\hline aspectl & 16211.00 & 71.19 & 69.76 & 0.78 & $0.731-0.817$ \\
\hline aspect2 & 15317.00 & 68.93 & 70.24 & 0.73 & $0.677-0.769$ \\
\hline uslope1 & 43.7 & 64.97 & 77.07 & 0.76 & $0.712-0.800$ \\
\hline uslope2 & 65.50 & 68.93 & 69.27 & 0.75 & $0.706-0.795$ \\
\hline dslopel & 25.917 & 74.58 & 63.41 & 0.75 & $0.699-0.789$ \\
\hline dslope2 & 19.286 & 63.84 & 75.12 & 0.70 & $0.646-0.741$ \\
\hline w1 & 21.00 & 59.32 & 55.61 & 0.60 & $0.553-0.654$ \\
\hline w2 & 17.00 & 53.11 & 57.07 & 0.55 & $0.498-0.600$ \\
\hline h1 & 365813.00 & 90.40 & 67.32 & 0.89 & $0.849-0.915$ \\
\hline h2 & 241.875 & 74.58 & 83.41 & 0.84 & $0.804-0.879$ \\
\hline divel & 297.25 & 81.36 & 70.24 & 0.81 & $0.763-0.844$ \\
\hline dive2 & 184.50 & 72.32 & 80.98 & 0.82 & $0.781-0.860$ \\
\hline path1 & 23.29 & 70.06 & 68.29 & 0.75 & $0.701-0.791$ \\
\hline path2 & 26473.00 & 64.97 & 64.88 & 0.69 & $0.639-0.734$ \\
\hline mslew 1 & 87.50 & 71.75 & 71.22 & 0.79 & $0.740-0.825$ \\
\hline mslew2 & 92.50 & 66.67 & 76.10 & 0.76 & $0.710-0.798$ \\
\hline slew1 & 48.821 & 64.97 & 71.71 & 0.71 & $0.666-0.759$ \\
\hline slew2 & 70563.00 & 69.49 & 66.34 & 0.73 & $0.684-0.775$ \\
\hline aplhf & 1.40 & 69.49 & 70.24 & 0.76 & $0.712-0.800$ \\
\hline plareal & 1189.75 & 85.88 & 80.49 & 0.90 & $0.870-0.931$ \\
\hline p2area1 & 751.875 & 83.05 & 83.90 & 0.90 & $0.865-0.928$ \\
\hline aspect11 & 20.15 & 61.02 & 74.15 & 0.71 & $0.658-0.751$ \\
\hline aspect21 & 19147.00 & 61.02 & 72.20 & 0.71 & $0.659-0.753$ \\
\hline uslope11 & 47125.00 & 61.58 & 65.37 & 0.69 & $0.643-0.738$ \\
\hline uslope21 & 47583.00 & 64.41 & 77.56 & 0.73 & $0.679-0.771$ \\
\hline dslope11 & 36.25 & 61.02 & 66.83 & 0.68 & $0.627-0.723$ \\
\hline dslope21 & 32214.00 & 61.58 & 61.46 & 0.67 & $0.624-0.721$ \\
\hline w11 & 10.00 & 59.32 & 66.83 & 0.65 & $0.597-0.695$ \\
\hline w21 & 8.00 & 63.28 & 59.02 & 0.63 & $0.577-0.677$ \\
\hline h11 & 231.75 & 85.31 & 71.22 & 0.89 & $0.849-0.915$ \\
\hline h21 & 161.25 & 74.58 & 83.41 & 0.84 & $0.804-0.879$ \\
\hline path11 & 34784.00 & 62.15 & 70.24 & 0.70 & $0.656-0.750$ \\
\hline path21 & 36901.00 & 61.02 & 60.00 & 0.64 & $0.590-0.688$ \\
\hline Fisher1.0 & 90.49 & 88.14 & 93.17 & 0.96 & $0.929-0.973$ \\
\hline
\end{tabular}

The AUROC was greater than 0.85 for 11 parameters. The parameter that achieved the best results was Fisher1.0 with AUR OC 0.96 , $88.14 \%$ sensitivity and specificity of $93.17 \%$

Table 3: Pairwise comparison of ROC curves

\begin{tabular}{lcccccccccc}
\hline & KC score & KC mod & KC NI & plarea & p2area & h1 & plareal & p2areal & h11 & Fisher1.0 \\
\hline CRF & 0.1522 & 0.5052 & 0.2405 & 0.2141 & 0.2878 & 0.785 & 0.5144 & 0.642 & 0.785 & $<0.0001$ \\
KC score & & 0.0102 & 0.0813 & 0.9883 & 0.6149 & 0.0274 & 0.4423 & 0.1253 & 0.0274 & $<0.0001$ \\
KC mod & & & 0.1075 & 0.4032 & 0.5185 & 0.2228 & 0.9317 & 0.7834 & 0.2228 & $<0.0001$ \\
KC NI & & & & 0.7542 & 0.9443 & 0.0677 & 0.6515 & 0.2875 & 0.0677 & $<0.0001$ \\
plarea & & & & & 0.7343 & 0.0058 & 0.0093 & 0.3164 & 0.0058 & 0.0001 \\
p2area & & & & & & 0.1489 & 0.7047 & 0.0448 & 0.1489 & 0.0003 \\
h1 & & & & & & & 0.1258 & 0.4156 & 10.000 & $<0.0001$ \\
plareal & & & & & & & & 0.7773 & 0.1258 & $<0.0001$ \\
p2areal & & & & & & & & & 0.4156 & $<0.0001$ \\
h11 & & & & & & & & & & $<0.0001$ \\
\hline
\end{tabular}




\section{DISCUSSION}

Corneal hydration, corneal thickness regional variation and collagen fibril orientation and distribution determine corneal biomechanics. ${ }^{9-12}$ A dditionally, the variation in biomechanical measurements may be due to age. ${ }^{13}$ Therefore, both groups consisted of patients of similar ages.

Keratoconic corneas should applanate slightly earlier and respond to a slightly lower rate of air pressure, which has also been reported in forme fruste keratoconus. ${ }^{5}$ Therefore, for corneas with keratoconus, lower values were found for most parameters. ${ }^{3,14,15}$ H owever, our data show ed that some parameters derived from the waveform signal; i.e. path 1, 2, 11, 21 and aplhf, were higher in patients with keratoconus. What makes these parameters higher in keratoconus? These parameters are related either to the absolute value of the path lengths around the peaks (path 1 , 11,2 and 21 ) or to the irregularity of the waveform region between the peaks (aplhf). Multiple oscillations of the waveform may reflect the characteristics of an ectatic cornea. ${ }^{5}$ Our study confirms the results for this condition, and should be taken into consideration in future clinical evaluations.

In terms of the pressure-derived parameters, the $\mathrm{CRF}$ was better than $\mathrm{CH}$, with $\mathrm{AUC} C \mathrm{CRF}=0.891$ compared to $A \cup C \mathrm{CH}=0.841$. These results confirm those of a previous study ${ }^{16}$ and may be related to findings that suggest that the CRF correlates best with the optical aberrations of keratoconus. ${ }^{17}$

A lthough $\mathrm{CH}$ and the $\mathrm{CRF}$ were significantly different between the two groups, there was an overlap, which limits the use of these parameters in isolation, as described previously. ${ }^{3,18}$ The parameters related to the signal waveform may better distinguish keratoconus from normal corneas than those derived from pressure parameters (L uce D. ORA waveform analysis and beyond. Presented at the A merican Society of Cataract and Refractive Surgery A nnual M eeting, A pril 3 to 8, 2009; San Francisco, California).

Our data demonstrate that the parameters derived from the areas under the first and second peaks were better than the traditional parameters. ${ }^{19} \mathrm{~W}$ ith A U C s of 0.915 and 0.910 , they exceeded the parameters $\mathrm{CH}$ and CRF (AUCS 0.841 and 0.891 respectively). Interestingly, the $\mathrm{KMI}$ and $\mathrm{KM} P$ indices achieved results similar to those derived from the areas under the first and second peaks, with A U C s of 0.910 and 0.915 respectively.

Of all parameters, the best was the Fisher 1.0. Its AUC of 0.955 exceeded those of either the pressure- or singlewaveform-derived parameters as well as from the K M I and KMP. Why does this parameter provide such a high combined sensitivity and specificity? This increase in the
A UC suggests that among the ORA parameters; there are some that do not clearly differentiate keratoconus from normal corneas when used independently, the performance of which are improved significantly when combined with other parameters. ${ }^{20}$ Figure 1 shows the ROC curves of the Fisher 1.0, p2area, which is the best parameter-derived signal, and CRF, which is the best parameter derived from the pressure. Figure 2 shows the difference between the result obtained with the Fisher 1.0 and $\mathrm{CH}$, which is the classic parameter.

The parameters of the ORA evolve. Firstly, the pressure derivatives, which consider the biological and biomechanical properties in addition to the geometric features (topography and pachymetry). From the signal curve, we noted that these data relevant provided information. Here the parameters were derived from the signal waveform, in particular the plarea and p2area. This develops further with $\mathrm{KMI}$ and $K M P$, but gives similar results.

Enhanced assessment was achieved with the Fisher 1.0, which is an analysis of the combination of all parameters.

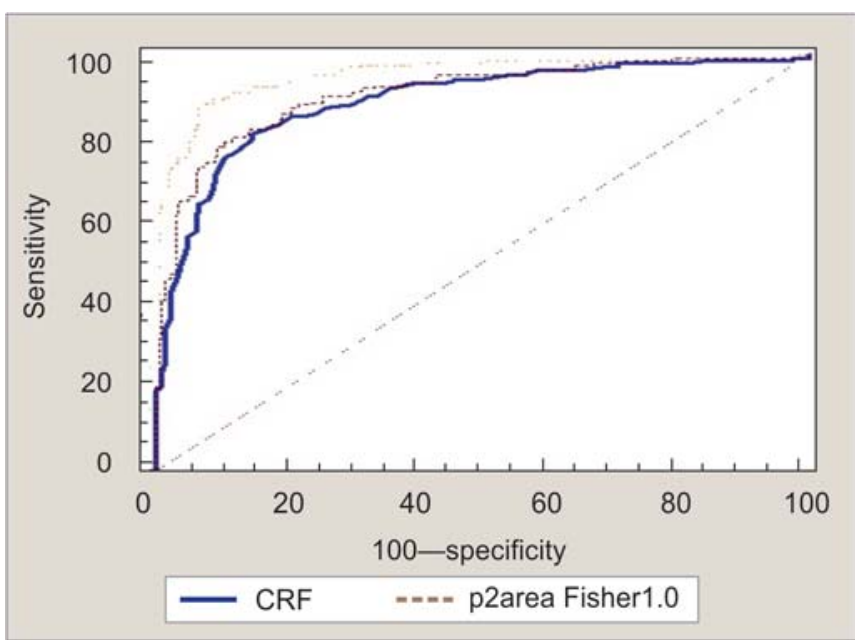

Fig. 1: Combined ROC curves for CRF, p2area and Fisher1.0

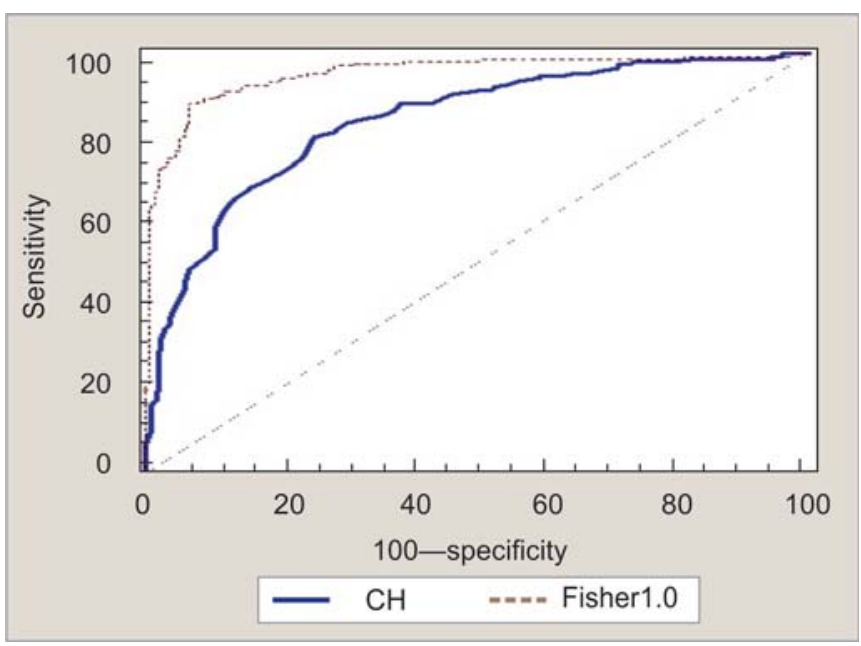

Fig. 2: Combined ROC curves for $\mathrm{CH}$ and Fisher1.0 
Future studies of this combined analysis in other populations should be performed.

The English in this document has been checked by at least two professional editors, both native speakers of English. For a certificate, please see: http:// www.textcheck.com/certificate/Em2DA G

\section{REFERENCES}

1. Dupps WJ J r, W ilson SE. B iomechanics and wound healing in the cornea. Exp Eye Res 2006;83:709-20.

2. Luce DA. Determining in vivo biomechanical properties of the cornea with an ocular response analyzer.J Cataract R efract Surg 2005;31:156-62.

3. Fontes BM, Ambrosio R Jr, et al. A bility of corneal biomechanical metrics and anterior segment data in the differentiation of keratoconus and healthy corneas. Arq Bras Oftalmol 2010;73:333-37.

4. Fontes BM, A mbrosio R J r, V elarde GC, Nose W. Corneal biomechanical evaluation in healthy thin corneas compared with matched keratoconus cases. A rq Bras Oftal mol 2011;74:13-16.

5. Kerautret J, Colin J, Touboul D, Roberts C. Biomechanical characteristics of the ectatic cornea. J Cataract Refract Surg 2008:34:510-13.

6. Alio J L, Shabayek M H. Corneal higher order aberrations: A method to grade keratoconus. J Refract Surg 2006;22:539-45.

7. DeL ong ER, D eL ong DM , Clarke-Pearson DL. Comparing the areas under two or more correlated receiver operating characteristic curves: A nonparametric approach. Biometrics 1988:44:837-45.

8. M CN eil BJ , H anley J A. Statistical approaches to the analysis of receiver operating characteristic (ROC) curves. M ed Decis Making 1984;4:137-50.

9. A nderson K, El-Sheikh A, N ewson T. A pplication of structural analysis to the mechanical behaviour of the cornea. J R Soc Interface 2004;1:3-15.

10. Luz A, U rsulio M, Castaneda D, A mbrosio R J r. Corneal thickness progression from the thinnest point to the limbus: Study based on a normal and a keratoconus population to create reference values. Arq B ras Oftalmol 2006;69:579-83.

11. A mbrosio R J r, A lonso RS, L uz A, Coca V elarde L G. Cornealthickness spatial profile and corneal-volume distribution: Tomographic indices to detect keratoconus. J Cataract R efract Surg 2006;32:1851-59.

12. Boote $C, H$ ayes $S, A$ bahussin $M$, M eek K M . M apping collagen organization in the human cornea: L eft and right eyes are structurally distinct. Invest O phthal mol V is Sci 2006;47:901-08.

13. Touboul D, Roberts $C$, K erautret J, et al. Correlations between corneal hysteresis, intraocular pressure and corneal central pachymetry. I Cataract Refract Surg 2008;34:616-22.

14. Fontes BM, A mbrosio R Jr, Velarde GC, Nose W. Ocular response analyzer measurements in keratoconus with normal central corneal thickness compared with matched normal control eyes. J Refract Surg 2011;27:209-15.

15. Touboul D, B enard A, M ahmoud A M , et al. Early biomechanical keratoconus pattern measured with an ocular response analyzer: curve analysis. J Cataract R efract Surg 2011;37:2144-50.

16. Mikielewicz M, Kotliar K, Barraquer RI, M ichael R. Air-pulse corneal applanation signal curve parameters for the characterisation of keratoconus. B J J Ophthalmol 2011;95:793-98.

17. Pinero DP, Alio J L, B arraquer RI, et al. Corneal biomechanics, refraction, and corneal aberrometry in keratoconus: an integrated study. Invest Ophthalmol V is Sci 2010;51:1948-55.

18. Fontes BM, A mbrosio R J r, Jardim D, et al. Corneal biomechanical metrics and anterior segment parameters in mild keratoconus. Ophthalmology 2010;117:673-79.

19. Luz A, Fontes B M , Lopes B, Ramos I, Schor P, A mbroso R J r. ORA waveform-derived biomechanical parameters to distinguish normal from keratoconic eyes. A rq B ras Oftalmol (article under review).

20. Guyon I, Elisseeff A. An introduction to variable and feature selection. J M achine Learning Res 2003;3:1157-82.

\section{ABOUT THE AUTHORS}

\section{Allan Luz (Corresponding Author)}

Department of Ophthalmology, Federal University of São Paulo Brazil, e-mail: dr.allanluz@gmail.com

\section{Bruno Fontes}

Department of O phthalmology, Federal U niversity of São Paulo, B razil

\section{Isaac C Ramos}

Department of Ophthalmology, Rio de J aneiro Corneal Tomography and Biomechanics Study Group, RJ, B razil

\section{Bernardo Lopes}

Department of Ophthal mology, Rio de J aneiro Corneal Tomography and Biomechanics Study Group, RJ, B razil

\section{Fernando Correia}

Department of Ophthalmology, Rio de J aneiro Corneal Tomography and B iomechanics Study Group, RJ, B razil

\section{Paulo Schor}

Department of Ophthalmology, Federal U niversity of São Paulo, B razil

\section{Renato Ambrósio Jr}

Department of Ophthalmology, Federal U niversity of São Paulo, B razil 